Turk. J. Math. Comput. Sci.

12(2)(2020) 76-85

(C) MatDer

DOI : $10.47000 /$ tjmcs. 715052

\title{
On The Norms of Another Form of $r$-Circulant Matrices with The Hyper-Fibonacci and Lucas Numbers
}

\author{
Mustafa BahşI $^{1, *}$ (D), Süleyman SolaK ${ }^{2}$ (D) \\ ${ }^{1}$ Department of Mathematics and Science Education, Faculty of Education, Aksaray University, 68100, Aksaray, Turkey. \\ ${ }^{2}$ Department of Mathematics and Science Education, Faculty of Education, N.E. University, 42090, Konya, Turkey.
}

Received: 05-04-2020 • Accepted: 05-07-2020

Aвstract. In this paper, we compute the spectral norms of $r$ - circulant matrices with the hyper-Fibonacci and hyper-Lucas numbers of the forms $F_{r}=\operatorname{Circ}-r\left(F_{k}^{(0)}, F_{k}^{(1)}, \ldots, F_{k}^{(n-1)}\right), L_{r}=\operatorname{Circ}-r\left(L_{k}^{(0)}, L_{k}^{(1)}, \ldots, L_{k}^{(n-1)}\right)$ and their Hadamard and Kronecker products. For this, we firstly compute the spectral and Euclidean norms of circulant matrices of the forms $F=\operatorname{Circ}\left(F_{k}^{(0)}, F_{k}^{(1)}, \ldots, F_{k}^{(n-1)}\right)$ and $L=\operatorname{Circ}\left(L_{k}^{(0)}, L_{k}^{(1)}, \ldots, L_{k}^{(n-1)}\right)$. Moreover, we give some examples related to special cases of our results.

2010 AMS Classification: 15A60, 15B05, 15B36, 11B39

Keywords: Circulant matrix, $r$-circulant matrix, Hyper- Fibonacci numbers, Hyper-Lucas numbers, Euclidean norm, Spectral norm.

\section{INTRODUCTION}

The circulant matrices and $r$ - circulant matrices are closely related to signal processing, coding theory and many other areas [1,10,11]. An $n \times n r$-circulant matrix $C_{r}$ is of the form

$$
C_{r}=\left[\begin{array}{cccccc}
c_{0} & c_{1} & c_{2} & \ldots & c_{n-2} & c_{n-1} \\
r c_{n-1} & c_{0} & c_{1} & \ldots & c_{n-3} & c_{n-2} \\
r c_{n-2} & r c_{n-1} & c_{0} & \ldots & c_{n-4} & c_{n-3} \\
\vdots & \vdots & \vdots & & \vdots & \vdots \\
r c_{1} & r c_{2} & r c_{3} & \ldots & r c_{n-1} & c_{0}
\end{array}\right] .
$$

When we take $r=1$, the matrix $C_{1}=C$ is called a circulant matrix. For brevity, we denote the matrices $C_{r}$ and $C_{1}$ as $C_{r}=\operatorname{Circ}-r\left(c_{0}, c_{1}, \ldots, c_{n-1}\right)$ and $C=\operatorname{Circ}\left(c_{0}, c_{1}, \ldots, c_{n-1}\right)$, respectively. If $A$ and $B$ are circulant matrices then they are normal, their inverses (if any), conjugate transposes, sums and products are also circulant [8]. The eigenvalues of $C$ are

$$
\underset{0 \leq m \leq n-1}{\lambda_{m}}=\sum_{k=0}^{n-1} c_{k} w^{-m k}
$$

where $w=e^{\frac{2 \pi i}{n}}$ and $i=\sqrt{-1}[8,14]$.

*Corresponding Author

Email addresses: mhvbahsi@yahoo.com (M. Bahşi), ssolak42@yahoo.com (S. Solak)

This paper is the extended form of the talk entitled "On the norms of another form of $r$ - circulant matrices with the hyper-Fibonacci and Lucas numbers" presented in the "International Conference on Mathematics and Mathematics Education" (ICMME-2016) at Firat University, Turkey, 12-14 May 2016. 
The circulant matrices and $r$-circulant matrices have been scientific research area in the recent past decades. Especially, the norms of circulant matrices with special elements such as Fibonacci or Fibonacci like numbers have been investigated extensively $[2,3,5,6,15-19,21-25]$. Shen and Cen [21] derived upper and lower bounds for the spectral norms of $r$-circulant matrices in the forms $A=C_{r}\left(F_{0}, F_{1}, \ldots, F_{n-1}\right)$ and $B=C_{r}\left(L_{0}, L_{1}, \ldots, L_{n-1}\right)$. Tuğlu and Kızllateş [18] studied norms of circulant and $r$-circulant matrices involving harmonic Fibonacci and hyperharmonic Fibonacci numbers. Türkmen and Gökbaş [24] found some bound estimations for the spectral norm of $r$-circulant matrices with Pell and Pell-Lucas numbers. In [5], the authors computed spectral norms of circulant matrices in the forms $F=\operatorname{Circ}\left(F_{0}^{(k)}, F_{1}^{(k)}, \ldots, F_{n-1}^{(k)}\right), L=\operatorname{Circ}\left(L_{0}^{(k)}, L_{1}^{(k)}, \ldots, L_{n-1}^{(k)}\right)$ and $r$-circulant matrices in the forms $F_{r}=\operatorname{Circ}-r\left(F_{0}^{(k)}\right.$, $\left.F_{1}^{(k)}, \ldots, F_{n-1}^{(k)}\right), L_{r}=\operatorname{Circ}-r\left(L_{0}^{(k)}, L_{1}^{(k)}, \ldots, L_{n-1}^{(k)}\right)$, where $F_{n}^{(k)}$ and $L_{n}^{(k)}$ denote the hyper-Fibonacci and hyper-Lucas numbers, respectively.

In this research, we establish some bounds for the spectral norms of $r$-circulant matrices with the hyper-Fibonacci and hyper-Lucas numbers of the forms $F_{r}=\operatorname{Circ}-r\left(F_{k}^{(0)}, F_{k}^{(1)}, \ldots, F_{k}^{(n-1)}\right), L_{r}=\operatorname{Circ}-r\left(L_{k}^{(0)}, L_{k}^{(1)}, \ldots, L_{k}^{(n-1)}\right)$ and their Hadamard and Kronecker products. For this, we firstly compute the spectral and Euclidean norms of circulant matrices of the forms $F=\operatorname{Circ}\left(F_{k}^{(0)}, F_{k}^{(1)}, \ldots, F_{k}^{(n-1)}\right)$ and $L=\operatorname{Circ}\left(L_{k}^{(0)}, L_{k}^{(1)}, \ldots, L_{k}^{(n-1)}\right)$. We use some relations concerning the spectral norm, Euclidean norm, row norm, column norm. Moreover, we give some examples related to special cases of our results.

\section{Preliminaries}

The Fibonacci numbers are defined by the recurrence relation: $F_{n+1}=F_{n}+F_{n-1}(n \geq 1), F_{0}=0$ and $F_{1}=1$. Similarly, the Lucas numbers are defined by $L_{n+1}=L_{n}+L_{n-1} \quad(n \geq 1), L_{0}=2$ and $L_{1}=1$. Fibonacci and Lucas numbers have many generalizations [7, 9, 20]. In [9], Dil and Mezö introduced two concepts as hyper - Fibonacci numbers and hyper - Lucas numbers. These concepts are defined as

$$
F_{n}^{(k)}=\sum_{s=0}^{n} F_{s}^{(k-1)}, \text { with } F_{n}^{(0)}=F_{n}, F_{0}^{(k)}=0 \text { and } F_{1}^{(k)}=1
$$

and

$$
L_{n}^{(k)}=\sum_{s=0}^{n} L_{s}^{(k-1)}, \text { with } L_{n}^{(0)}=L_{n}, L_{0}^{(k)}=2, L_{1}^{(k)}=2 k+1 .
$$

The hyper-Fibonacci and the hyper-Lucas numbers have the recurrence relations $F_{n}^{(k)}=F_{n-1}^{(k)}+F_{n}^{(k-1)}$ and $L_{n}^{(k)}=$ $L_{n-1}^{(k)}+L_{n}^{(k-1)}$, respectively. Also, $F_{n}^{(k)}$ and $L_{n}^{(k)}$ have the following more explicit forms when $k=1,2,3$ or $n=2,3$.

$$
\begin{gathered}
F_{n}^{(1)}=F_{n+2}-1, \quad F_{n}^{(2)}=F_{n+4}-n-3 \text { and } F_{n}^{(3)}=F_{n+6}-\frac{n^{2}+7 n+16}{2}, \\
L_{n}^{(1)}=L_{n+2}-1, \quad L_{n}^{(2)}=L_{n+4}-n-5 \text { and } L_{n}^{(3)}=L_{n+6}-\frac{n^{2}+11 n+32}{2} . \\
F_{2}^{(n)}=n+1, \quad F_{3}^{(n)}=\frac{n^{2}+3 n+4}{2} \text { and } L_{2}^{(n)}=n^{2}+2 n+3 .
\end{gathered}
$$

In [4], the authors defined hyper-Horadam numbers and studied their some properties. Also, they gave the following formulas related to sums of hyper - Fibonacci and hyper - Lucas numbers

$$
\sum_{s=0}^{r} F_{n}^{(s)}=F_{n+1}^{(r)}-F_{n-1}
$$

and

$$
\sum_{s=0}^{s} L_{n}^{(s)}=L_{n+1}^{(r)}-L_{n-1}
$$

For more information related to hyper - Fibonacci numbers see $[4,7,9]$.

Now we give some definitions and lemmas related to our study. 
Definition 2.1. Let $A=\left(a_{i j}\right)$ be any $m \times n$ matrix. The Euclidean norm of $A$ is

$$
\|A\|_{E}=\sqrt{\left(\sum_{i=1}^{m} \sum_{j=1}^{n}\left|a_{i j}\right|^{2}\right) .}
$$

Definition 2.2. Let $A=\left(a_{i j}\right)$ be any $m \times n$ matrix. The spectral norm of $A$ is

$$
\|A\|_{2}=\sqrt{\max _{i} \lambda_{i}\left(A^{H} A\right)},
$$

where $\lambda_{i}\left(A^{H} A\right)$ are eigenvalues of $A^{H} A$ and $A^{H}$ is conjugate transpose of $A$.

There are two well known relations between Euclidean norm and spectral norm as the following:

$$
\begin{aligned}
& \frac{1}{\sqrt{n}}\|A\|_{E} \leq\|A\|_{2} \leq\|A\|_{E} \\
& \|A\|_{2} \leq\|A\|_{E} \leq \sqrt{n}\|A\|_{2} .
\end{aligned}
$$

Definition 2.3 ( [13]). Let $A=\left(a_{i j}\right)$ and $B=\left(b_{i j}\right)$ be $m \times n$ matrices.Then their Hadamard product A $\circ \mathrm{B}$ is defined

$$
A \circ B=\left[a_{i j} b_{i j}\right] .
$$

Definition 2.4 ( [13]). Let $A=\left(a_{i j}\right)$ and $B=\left(b_{i j}\right)$ be $m \times n$ and $p \times r$ matrices, respectively.Then their Kronecker product $\mathrm{A} \otimes \mathrm{B}$ is defined

$$
A \otimes B=\left[a_{i j} B\right] .
$$

Lemma 2.5 ( [13]). Let $A$ and $B$ be two $m \times n$ matrices. Then we have

$$
\|A \circ B\|_{2} \leq\|A\|_{2}\|B\|_{2} .
$$

Lemma 2.6 ([13]). Let $A$ and $B$ be two $m \times n$ matrices. Then we have

$$
\|A \circ B\|_{2} \leq r_{1}(A) c_{1}(B)
$$

where $r_{1}(A)=\max _{1 \leq i \leq m} \sqrt{\sum_{j=1}^{n}\left|a_{i j}\right|^{2}}$ and $c_{1}(B)=\max _{1 \leq j \leq n} \sqrt{\sum_{i=1}^{m}\left|b_{i j}\right|^{2}}$.

Lemma 2.7 ( [13]). Let $A$ and $B$ be two $m \times n$ matrices. Then we have

$$
\|A \otimes B\|_{2}=\|A\|_{2}\|B\|_{2} .
$$

Lemma 2.8 ([12]). Let $A$ be an $n \times n$ matrix with eigenvalues $\lambda_{1}, \lambda_{2}, \ldots, \lambda_{n}$. Then, $A$ is a normal matrix if and only if the eigenvalues of $A^{H} A$ are $\left|\lambda_{1}\right|^{2},\left|\lambda_{2}\right|^{2}, \ldots,\left|\lambda_{n}\right|^{2}$.

\section{Main Results}

Theorem 3.1. The spectral norm of the matrix $F=\operatorname{Circ}\left(F_{k}^{(0)}, F_{k}^{(1)}, \ldots, F_{k}^{(n-1)}\right)$ is

$$
\|F\|_{2}=F_{k+1}^{(n-1)}-F_{k-1} \text {. }
$$

Proof. Since the circulant matrix $F$ is normal, its spectral norm is equal to its spectral radius. Furthermore, by considering $F$ is irreducible and its entries are nonnegative, we have that the spectral radius (or spectral norm) of the matrix $F$ is equal to its Perron root. We select an $n$-dimensional column vector $v=(1,1, \ldots, 1)^{T}$, then

$$
F v=\left(\sum_{s=0}^{n-1} F_{k}^{(s)}\right) v .
$$

Obviously, $\sum_{s=0}^{n-1} F_{k}^{(s)}$ is an eigenvalue of $F$ associated with $v$ and it is the Perron root of $F$. Hence, by (2.2) we have

$$
\|F\|_{2}=\sum_{s=0}^{n-1} F_{k}^{(s)}=F_{k+1}^{(n-1)}-F_{k-1} .
$$


This completes the proof.

Example 3.2. Theorem 3.1 and the equations in (2.1) yield

$$
\|F\|_{2}=\left\{\begin{array}{cc}
0, & \text { if } k=0, \\
n, & \text { if } k=1, \\
\frac{n^{2}+n}{2}, & \text { if } k=2 .
\end{array}\right.
$$

Corollary 3.3. Euclidean norm of the matrix $F=\operatorname{Circ}\left(F_{k}^{(0)}, F_{k}^{(1)}, \ldots, F_{k}^{(n-1)}\right)$ holds

$$
F_{k+1}^{(n-1)}-F_{k-1} \leq\|F\|_{E} \leq \sqrt{n}\left(F_{k+1}^{(n-1)}-F_{k-1}\right) .
$$

Proof. The proof is trivial from Theorem 3.1 and the relation between spectral norm and Euclidean norm in (2.4).

Corollary 3.4. We have

$$
\frac{1}{\sqrt{n}}\left(F_{k+1}^{(n-1)}-F_{k-1}\right) \leq \sqrt{\sum_{s=0}^{n-1}\left(F_{k}^{(s)}\right)^{2}} \leq F_{k+1}^{(n-1)}-F_{k-1} .
$$

Proof. This follows from the definition of Euclidean norm and Corollary 3.3.

Theorem 3.5. The spectral norm of the matrix $L=\operatorname{Circ}\left(L_{k}^{(0)}, L_{k}^{(1)}, \ldots, L_{k}^{(n-1)}\right)$ is

$$
\|L\|_{2}=L_{k+1}^{(n-1)}-L_{k-1} \text {. }
$$

Proof. This theorem can be proved by using a similar method to method of the proof of Theorem 3.1.

Example 3.6. Theorem 3.5 and the equations in (2.1) yield

$$
\|L\|_{2}=\left\{\begin{array}{cc}
2 n, & \text { if } k=0 \\
n^{2}+2 n+1, & \text { if } k=1
\end{array}\right.
$$

Corollary 3.7. We have

$$
L_{k+1}^{(n-1)}-L_{k-1} \leq\|L\|_{E} \leq \sqrt{n}\left(L_{k+1}^{(n-1)}-L_{k-1}\right) .
$$

Proof. Theorem 3.5 and the relation between spectral norm and Euclidean norm in (2.4) immediately yield desired result.

Corollary 3.8. Sum of squares of hyper-Lucas numbers holds

$$
\frac{1}{\sqrt{n}}\left(L_{k+1}^{(n-1)}-L_{k-1}\right) \leq \sqrt{\sum_{s=0}^{n-1}\left(L_{k}^{(s)}\right)^{2}} \leq L_{k+1}^{(n-1)}-L_{k-1} .
$$

Proof. From the definition of Euclidean norm and Corollary 3.7, desired result is obtained.

Corollary 3.9. The spectral norm of the Hadamard product of $F=\operatorname{Circ}\left(F_{k}^{(0)}, F_{k}^{(1)}, \ldots, F_{k}^{(n-1)}\right)$ and $L=\operatorname{Circ}\left(L_{k}^{(0)}, L_{k}^{(1)}, \ldots, L_{k}^{(n-1)}\right)$ satisfies

$$
\|F \circ L\|_{2} \leq\left(F_{k+1}^{(n-1)}-F_{k-1}\right)\left(L_{k+1}^{(n-1)}-L_{k-1}\right) .
$$

Proof. Since $\|F \circ L\|_{2} \leq\|F\|_{2}\|L\|_{2}$ desired result is trivial.

Corollary 3.10. The spectral norm of the Kronecker product of $F=\operatorname{Circ}\left(F_{k}^{(0)}, F_{k}^{(1)}, \ldots, F_{k}^{(n-1)}\right)$ and $L=\operatorname{Circ}\left(L_{k}^{(0)}, L_{k}^{(1)}, \ldots, L_{k}^{(n-1)}\right)$ satisfies

$$
\|F \otimes L\|_{2}=\left(F_{k+1}^{(n-1)}-F_{k-1}\right)\left(L_{k+1}^{(n-1)}-L_{k-1}\right) .
$$

Proof. Since $\|F \otimes L\|_{2}=\|F\|_{2}\|L\|_{2}$ we get the desired result.

Theorem 3.11. Let $F_{r}=\operatorname{Circ}-r\left(F_{k}^{(0)}, F_{k}^{(1)}, \ldots, F_{k}^{(n-1)}\right)$ be an $r$-circulant matrix.

i) If $|r| \geq 1$, then

$$
\frac{1}{\sqrt{n}}\left(F_{k+1}^{(n-1)}-F_{k-1}\right) \leq\left\|F_{r}\right\|_{2} \leq|r|\left(F_{k+1}^{(n-1)}-F_{k-1}\right)^{2}
$$


ii) If $|r|<1$, then

$$
\frac{|r|}{\sqrt{n}}\left(F_{k+1}^{(n-1)}-F_{k-1}\right) \leq\left\|F_{r}\right\|_{2} \leq \sqrt{n}\left(F_{k+1}^{(n-1)}-F_{k-1}\right) .
$$

Proof. Since the matrix $F_{r}$ is of the form

$$
F_{r}=\left[\begin{array}{cccccc}
F_{k}^{(0)} & F_{k}^{(1)} & F_{k}^{(2)} & \cdots & F_{k}^{(n-2)} & F_{k}^{(n-1)} \\
r F_{k}^{(n-1)} & F_{k}^{(0)} & F_{k}^{(1)} & \cdots & F_{k}^{(n-3)} & F_{k}^{(n-2)} \\
\vdots & \vdots & \vdots & \ddots & \vdots & \vdots \\
r F_{k}^{(2)} & r F_{k}^{(3)} & r F_{k}^{(4)} & \cdots & F_{k}^{(0)} & F_{k}^{(1)} \\
r F_{k}^{(1)} & r F_{k}^{(2)} & r F_{k}^{(3)} & \cdots & r F_{k}^{(n-1)} & F_{k}^{(0)}
\end{array}\right]
$$

and from the definition of Euclidean norm, we have

$$
\left\|F_{r}\right\|_{E}=\sqrt{\sum_{s=0}^{n-1}(n-s)\left(F_{k}^{(s)}\right)^{2}+\sum_{s=0}^{n-1} s|r|^{2}\left(F_{k}^{(s)}\right)^{2}} .
$$

i) Since $|r| \geq 1$, (3.1) yields

$$
\left\|F_{r}\right\|_{E} \geq \sqrt{\sum_{s=0}^{n-1}(n-s)\left(F_{k}^{(s)}\right)^{2}+\sum_{s=0}^{n-1} s\left(F_{k}^{(s)}\right)^{2}}=\sqrt{n \sum_{s=0}^{n-1}\left(F_{k}^{(s)}\right)^{2}} \geq F_{k+1}^{(n-1)}-F_{k-1} .
$$

From (2.4)

Let $F_{r}$ be $F_{r}=B \circ C$, where

$$
\left\|F_{r}\right\|_{2} \geq \frac{1}{\sqrt{n}}\left(F_{k+1}^{(n-1)}-F_{k-1}\right)
$$

$$
B=\left[\begin{array}{cccccc}
1 & 1 & 1 & \cdots & 1 & 1 \\
r F_{k}^{(n-1)} & 1 & 1 & \cdots & 1 & 1 \\
\vdots & \vdots & \vdots & \ddots & \vdots & \vdots \\
r F_{k}^{(2)} & r F_{k}^{(3)} & r F_{k}^{(4)} & \cdots & 1 & 1 \\
r F_{k}^{(1)} & r F_{k}^{(2)} & r F_{k}^{(3)} & \cdots & r F_{k}^{(n-1)} & 1
\end{array}\right]
$$

and

$$
C=\left[\begin{array}{cccccc}
F_{k}^{(0)} & F_{k}^{(1)} & F_{k}^{(2)} & \cdots & F_{k}^{(n-2)} & F_{k}^{(n-1)} \\
1 & F_{k}^{(0)} & F_{k}^{(1)} & \cdots & F_{k}^{(n-3)} & F_{k}^{(n-2)} \\
\vdots & \vdots & \vdots & \ddots & \vdots & \vdots \\
1 & 1 & 1 & \cdots & F_{k}^{(0)} & F_{k}^{(1)} \\
1 & 1 & 1 & \cdots & 1 & F_{k}^{(0)}
\end{array}\right] .
$$

Then

$$
\begin{aligned}
r_{1}(B) & =\max _{1 \leq i \leq n} \sqrt{\sum_{j=1}^{n}\left|b_{i j}\right|^{2}}=\sqrt{\sum_{j=1}^{n}\left|b_{n j}\right|^{2}}=\sqrt{1+\sum_{s=1}^{n-1}|r|^{2}\left(F_{k}^{(s)}\right)^{2}} \\
& \leq|r| \sqrt{\sum_{s=0}^{n-1}\left(F_{k}^{(s)}\right)^{2}}
\end{aligned}
$$

and

$$
c_{1}(C)=\max _{1 \leq j \leq n} \sqrt{\sum_{i=1}^{n}\left|c_{i j}\right|^{2}}=\sqrt{\sum_{i=1}^{n}\left|c_{i n}\right|^{2}}=\sqrt{\sum_{s=0}^{n-1}\left(F_{k}^{(s)}\right)^{2}} .
$$

(3.1) and Lemma 2.3 yield

$$
\left\|F_{r}\right\|_{2} \leq r_{1}(B) c_{1}(C) \leq|r|\left(F_{k+1}^{(n-1)}-F_{k-1}\right)^{2} .
$$


Thus,

$$
\frac{1}{\sqrt{n}}\left(F_{k+1}^{(n-1)}-F_{k-1}\right) \leq\left\|F_{r}\right\|_{2} \leq|r|\left(F_{k+1}^{(n-1)}-F_{k-1}\right)^{2} .
$$

ii) Since $|r|<1$, (3.1) yields

$$
\begin{aligned}
\left\|F_{r}\right\|_{E} & =\sqrt{\sum_{s=0}^{n-1}(n-s)\left(F_{k}^{(s)}\right)^{2}+\sum_{s=0}^{n-1} s|r|^{2}\left(F_{k}^{(s)}\right)^{2}} \\
& \geq \sqrt{\sum_{s=0}^{n-1}(n-s)|r|^{2}\left(F_{k}^{(s)}\right)^{2}+\sum_{s=0}^{n-1} s|r|^{2}\left(F_{k}^{(s)}\right)^{2}} \\
& =\sqrt{n|r|^{2} \sum_{s=0}^{n-1}\left(F_{k}^{(s)}\right)^{2}} \geq|r|\left(F_{k+1}^{(n-1)}-F_{k-1}\right) .
\end{aligned}
$$

From (2.4)

$$
\left\|F_{r}\right\|_{2} \geq \frac{|r|}{\sqrt{n}}\left(F_{k+1}^{(n-1)}-F_{k-1}\right) .
$$

Now, let the matrices $F_{r}$ be $F_{r}=D \circ E$, where

$$
D=\left[\begin{array}{cccccc}
1 & 1 & 1 & \cdots & 1 & 1 \\
r & 1 & 1 & \cdots & 1 & 1 \\
\vdots & \vdots & \vdots & \ddots & \vdots & \vdots \\
r & r & r & \cdots & 1 & 1 \\
r & r & r & \cdots & r & 1
\end{array}\right]
$$

and

Then we compute $r_{1}(D)$ and $c_{1}(E)$ as

$$
E=\left[\begin{array}{cccccc}
F_{k}^{(0)} & F_{k}^{(1)} & F_{k}^{(2)} & \cdots & F_{k}^{(n-2)} & F_{k}^{(n-1)} \\
F_{k}^{(n-1)} & F_{k}^{(0)} & F_{k}^{(1)} & \cdots & F_{k}^{(n-3)} & F_{k}^{(n-2)} \\
\vdots & \vdots & \vdots & \ddots & \vdots & \vdots \\
F_{k}^{(2)} & F_{k}^{(3)} & F_{k}^{(4)} & \cdots & F_{k}^{(0)} & F_{k}^{(1)} \\
F_{k}^{(1)} & F_{k}^{(2)} & F_{k}^{(3)} & \cdots & F_{k}^{(n-1)} & F_{k}^{(0)}
\end{array}\right]
$$

$$
r_{1}(D)=\max _{1 \leq i \leq n} \sqrt{\sum_{j=1}^{n}\left|d_{i j}\right|^{2}}=\sqrt{n}
$$

and

$$
c_{1}(E)=\max _{1 \leq j \leq n} \sqrt{\sum_{i=1}^{n}\left|e_{i j}\right|^{2}}=\sqrt{\sum_{s=0}^{n-1}\left(F_{k}^{(s)}\right)^{2}} .
$$

Hence, from (3.1) and Lemma 2.3, we have

$$
\left\|F_{r}\right\|_{2} \leq r_{1}(B) c_{1}(E) \leq \sqrt{n}\left(F_{k+1}^{(n-1)}-F_{k-1}\right) .
$$

Thus,

$$
\frac{|r|}{\sqrt{n}}\left(F_{k+1}^{(n-1)}-F_{k-1}\right) \leq\left\|F_{r}\right\|_{2} \leq \sqrt{n}\left(F_{k+1}^{(n-1)}-F_{k-1}\right) .
$$

This completes the proof.

Example 3.12. By using Theorem 3.11 and the equations in (2.1), if $|r| \geq 1$, we have

$$
\left\|F_{r}\right\|_{2}=0 \text {, if } k=0 \text {, }
$$




$$
\begin{aligned}
\sqrt{n} & \leq\left\|F_{r}\right\|_{2} \leq|r| n^{2}, \text { if } k=1, \\
\frac{1}{\sqrt{n}}\left(\frac{n^{2}+n}{2}\right) & \leq\left\|F_{r}\right\|_{2} \leq|r|\left(\frac{n^{2}+n}{2}\right)^{2}, \text { if } k=2,
\end{aligned}
$$

and if $|r|<1$, we have

$$
\begin{gathered}
\left\|F_{r}\right\|_{2}=0 \text {, if } k=0, \\
|r| \sqrt{n} \leq\left\|F_{r}\right\|_{2} \leq n^{2} \sqrt{n} \text {, if } k=1, \\
\frac{|r|}{\sqrt{n}}\left(\frac{n^{2}+n}{2}\right) \leq\left\|F_{r}\right\|_{2} \leq \sqrt{n}\left(\frac{n^{2}+n}{2}\right), \text { if } k=2 .
\end{gathered}
$$

Theorem 3.13. Let $L_{r}=\operatorname{Circ}-r\left(L_{k}^{(0)}, L_{k}^{(1)}, \ldots, L_{k}^{(n-1)}\right)$ be an $r$-circulant matrix.

i) If $|r| \geq 1$, then

$$
\frac{1}{\sqrt{n}}\left(L_{k+1}^{(n-1)}-L_{k-1}\right) \leq\left\|L_{r}\right\|_{2} \leq|r|\left(L_{k+1}^{(n-1)}-L_{k-1}\right)^{2} .
$$

ii) If $|r|<1$, then

$$
\frac{|r|}{\sqrt{n}}\left(L_{k+1}^{(n-1)}-L_{k-1}\right) \leq\left\|L_{r}\right\|_{2} \leq \sqrt{n}\left(L_{k+1}^{(n-1)}-L_{k-1}\right) .
$$

Proof. Since the matrix $L_{r}$ is of the form

$$
L_{r}=\left[\begin{array}{cccccc}
L_{k}^{(0)} & L_{k}^{(1)} & L_{k}^{(2)} & \cdots & L_{k}^{(n-2)} & L_{k}^{(n-1)} \\
r L_{k}^{(n-1)} & L_{k}^{(0)} & L_{k}^{(1)} & \cdots & L_{k}^{(n-3)} & L_{k}^{(n-2)} \\
\vdots & \vdots & \vdots & \ddots & \vdots & \vdots \\
r L_{k}^{(2)} & r L_{k}^{(3)} & r L_{k}^{(4)} & \cdots & L_{k}^{(0)} & L_{k}^{(1)} \\
r L_{k}^{(1)} & r L_{k}^{(2)} & r L_{k}^{(3)} & \cdots & r L_{k}^{(n-1)} & L_{k}^{(0)}
\end{array}\right]
$$

and from the definition of Euclidean norm, we have

$$
\left\|L_{r}\right\|_{E}=\sqrt{\sum_{s=0}^{n-1}(n-s)\left(L_{k}^{(s)}\right)^{2}+\sum_{s=0}^{n-1} s|r|^{2}\left(L_{k}^{(s)}\right)^{2}} .
$$

i) Since $|r| \geq 1$, (3.2) yields

$$
\left\|L_{r}\right\|_{E} \geq \sqrt{\sum_{s=0}^{n-1}(n-s)\left(L_{k}^{(s)}\right)^{2}+\sum_{s=0}^{n-1} s\left(L_{k}^{(s)}\right)^{2}}=\sqrt{n \sum_{s=0}^{n-1}\left(L_{k}^{(s)}\right)^{2}} \geq L_{k+1}^{(n-1)}-L_{k-1} .
$$

From (2.3)

$$
\left\|L_{r}\right\|_{2} \geq \frac{1}{\sqrt{n}}\left(L_{k+1}^{(n-1)}-L_{k-1}\right) .
$$

Now, let the matrices $L_{r}$ be $L_{r}=B \circ C$, where

$$
B=\left[\begin{array}{cccccc}
1 & 1 & 1 & \cdots & 1 & 1 \\
r L_{k}^{(n-1)} & 1 & 1 & \cdots & 1 & 1 \\
\vdots & \vdots & \vdots & \ddots & \vdots & \vdots \\
r L_{k}^{(2)} & r L_{k}^{(3)} & r L_{k}^{(4)} & \cdots & 1 & 1 \\
r L_{k}^{(1)} & r L_{k}^{(2)} & r L_{k}^{(3)} & \cdots & r L_{k}^{(n-1)} & 1
\end{array}\right]
$$


and

$$
C=\left[\begin{array}{cccccc}
L_{k}^{(0)} & L_{k}^{(1)} & L_{k}^{(2)} & \ldots & L_{k}^{(n-2)} & L_{k}^{(n-1)} \\
1 & L_{k}^{(0)} & L_{k}^{(1)} & \ldots & L_{k}^{(n-3)} & L_{k}^{(n-2)} \\
\vdots & \vdots & \vdots & & \vdots & \vdots \\
1 & 1 & 1 & \ldots & L_{k}^{(0)} & L_{k}^{(1)} \\
1 & 1 & 1 & \ldots & 1 & L_{k}^{(0)}
\end{array}\right]
$$

Then we have

$$
\begin{aligned}
r_{1}(B) & =\max _{1 \leq i \leq n} \sqrt{\sum_{j=1}^{n}\left|b_{i j}\right|^{2}}=\sqrt{\sum_{j=1}^{n}\left|b_{n j}\right|^{2}}=\sqrt{1+\sum_{s=1}^{n-1}|r|^{2}\left(L_{k}^{(s)}\right)^{2}} \\
& \leq|r| \sqrt{\sum_{s=0}^{n-1}\left(L_{k}^{(s)}\right)^{2}}
\end{aligned}
$$

and

$$
c_{1}(C)=\max _{1 \leq j \leq n} \sqrt{\sum_{i=1}^{n}\left|c_{i j}\right|^{2}}=\sqrt{\sum_{i=1}^{n}\left|c_{i n}\right|^{2}}=\sqrt{\sum_{s=0}^{n-1}\left(L_{k}^{(s)}\right)^{2}} .
$$

Hence, from (3.2) and Lemma 2.3, we have

$$
\left\|L_{r}\right\|_{2} \leq r_{1}(B) c_{1}(C) \leq|r|\left(L_{k+1}^{(n-1)}-L_{k-1}\right)^{2} .
$$

Thus, we write

$$
\frac{1}{\sqrt{n}}\left(L_{k+1}^{(n-1)}-L_{k-1}\right) \leq\left\|L_{r}\right\|_{2} \leq|r|\left(L_{k+1}^{(n-1)}-L_{k-1}\right)^{2} .
$$

ii) Since $|r|<1$, (3.2) yields

$$
\begin{aligned}
\left\|L_{r}\right\|_{E} & =\sqrt{\sum_{s=0}^{n-1}(n-s)\left(L_{k}^{(s)}\right)^{2}+\sum_{s=0}^{n-1} s|r|^{2}\left(L_{k}^{(s)}\right)^{2}} \\
& \geq \sqrt{\sum_{s=0}^{n-1}(n-s)|r|^{2}\left(L_{k}^{(s)}\right)^{2}+\sum_{s=0}^{n-1} s|r|^{2}\left(L_{k}^{(s)}\right)^{2}} \\
& =\sqrt{n|r|^{2} \sum_{s=0}^{n-1}\left(L_{k}^{(s)}\right)^{2}} \geq|r|\left(L_{k+1}^{(n-1)}-L_{k-1}\right) .
\end{aligned}
$$

From (2.3)

Now, let the matrices $B$ and $C$ be as

$$
\left\|L_{r}\right\|_{2} \geq \frac{|r|}{\sqrt{n}}\left(L_{k+1}^{(n-1)}-L_{k-1}\right)
$$

$$
D=\left[\begin{array}{cccccc}
1 & 1 & 1 & \cdots & 1 & 1 \\
r & 1 & 1 & \cdots & 1 & 1 \\
\vdots & \vdots & \vdots & \ddots & \vdots & \vdots \\
r & r & r & \cdots & 1 & 1 \\
r & r & r & \cdots & r & 1
\end{array}\right]
$$

and

$$
E=\left[\begin{array}{cccccc}
L_{k}^{(0)} & L_{k}^{(1)} & L_{k}^{(2)} & \cdots & L_{k}^{(n-2)} & L_{k}^{(n-1)} \\
L_{k}^{(n-1)} & L_{k}^{(0)} & L_{k}^{(1)} & \cdots & L_{k}^{(n-3)} & L_{k}^{(n-2)} \\
\vdots & \vdots & \vdots & \ddots & \vdots & \vdots \\
L_{k}^{(2)} & L_{k}^{(3)} & L_{k}^{(4)} & \cdots & L_{k}^{(0)} & L_{k}^{(1)} \\
L_{k}^{(1)} & L_{k}^{(2)} & L_{k}^{(3)} & \cdots & L_{k}^{(n-1)} & L_{k}^{(0)}
\end{array}\right]
$$


That is, $L_{r}=B \circ C$. Then we obtain

and

$$
r_{1}(D)=\max _{1 \leq i \leq n} \sqrt{\sum_{j=1}^{n}\left|d_{i j}\right|^{2}}=\sqrt{n}
$$

(3.2) and Lemma 2.3 yield

$$
c_{1}(E)=\max _{1 \leq j \leq n} \sqrt{\sum_{i=1}^{n}\left|e_{i j}\right|^{2}}=\sqrt{\sum_{s=0}^{n-1}\left(L_{k}^{(s)}\right)^{2}} .
$$

$$
\left\|L_{r}\right\|_{2} \leq r_{1}(B) c_{1}(E) \leq \sqrt{n}\left(L_{k+1}^{(n-1)}-L_{k-1}\right) .
$$

Thus,

This completes the proof.

$$
\frac{|r|}{\sqrt{n}}\left(L_{k+1}^{(n-1)}-L_{k-1}\right) \leq\left\|L_{r}\right\|_{2} \leq \sqrt{n}\left(L_{k+1}^{(n-1)}-L_{k-1}\right) .
$$

Example 3.14. By using Theorem 3.13 and the equations in (2.1), if $|r| \geq 1$, we have

$$
\begin{aligned}
2 \sqrt{n} & \leq\left\|L_{r}\right\|_{2} \leq 4 n^{2}|r|, \quad \text { if } k=0, \\
\frac{1}{\sqrt{n}}\left(n^{2}+2 n+1\right) & \leq\left\|L_{r}\right\|_{2} \leq|r|\left(n^{2}+2 n+1\right)^{2}, \quad \text { if } k=1,
\end{aligned}
$$

and if $|r|<1$, we have

$$
\begin{aligned}
2 \sqrt{n}|r| & \leq\left\|L_{r}\right\|_{2} \leq 2 n \sqrt{n}, \quad \text { if } k=0, \\
\frac{|r|}{\sqrt{n}}\left(n^{2}+2 n+1\right) & \leq\left\|L_{r}\right\|_{2} \leq \sqrt{n}\left(n^{2}+2 n+1\right), \quad \text { if } k=1 .
\end{aligned}
$$

Corollary 3.15. The spectral norm of the Hadamard product of $F_{r}=\operatorname{Circ}-r\left(F_{k}^{(0)}, F_{k}^{(1)}, \ldots, F_{k}^{(n-1)}\right)$ and $L_{r}=\operatorname{Circ}-r\left(L_{k}^{(0)}, L_{k}^{(1)}, \ldots, L_{k}^{(n-1)}\right)$ holds

i) If $|r| \geq 1$, then

ii) If $|r|<1$, then

$$
\left\|F_{r} \circ L_{r}\right\|_{2} \leq|r|^{2}\left(F_{k+1}^{(n-1)}-F_{k-1}\right)^{2}\left(L_{k+1}^{(n-1)}-L_{k-1}\right)^{2} .
$$

$$
\left\|F_{r} \circ L_{r}\right\|_{2} \leq n\left(F_{k+1}^{(n-1)}-F_{k-1}\right)\left(L_{k+1}^{(n-1)}-L_{k-1}\right) .
$$

Proof. The proof is trivial since $\left\|F_{r} \circ L_{r}\right\|_{2} \leq\left\|F_{r}\right\|_{2}\left\|L_{r}\right\|_{2}$.

Corollary 3.16. The spectral norm of the Kronecker product of $F_{r}=\operatorname{Circ}-r\left(F_{k}^{(0)}, F_{k}^{(1)}, \ldots, F_{k}^{(n-1)}\right)$ and $L_{r}=\operatorname{Circ}-r\left(L_{k}^{(0)}, L_{k}^{(1)}, \ldots, L_{k}^{(n-1)}\right)$ holds

i) If $|r| \geq 1$, then

$$
\frac{1}{n}\left(F_{k+1}^{(n-1)}-F_{k-1}\right)\left(L_{k+1}^{(n-1)}-L_{k-1}\right) \leq\left\|F_{r} \otimes L_{r}\right\|_{2} \leq|r|^{2}\left(F_{k+1}^{(n-1)}-F_{k-1}\right)^{2}\left(L_{k+1}^{(n-1)}-L_{k-1}\right)^{2} .
$$

ii) If $|r|<1$, then

$$
\frac{|r|^{2}}{n}\left(F_{k+1}^{(n-1)}-F_{k-1}\right)\left(L_{k+1}^{(n-1)}-L_{k-1}\right) \leq\left\|F_{r} \otimes L_{r}\right\|_{2} \leq n\left(F_{n-1}^{(k+1)}\right)^{2}\left(L_{n-1}^{(k+1)}\right) .
$$

Proof. The proof is trivial since $\left\|F_{r} \otimes L_{r}\right\|_{2}=\left\|F_{r}\right\|_{2}\left\|L_{r}\right\|_{2}$.

\section{Conclusion}

In this study, we present some bounds for the spectral norms of a different form of $r$-circulant matrices with the hyper-Fibonacci and hyper-Lucas numbers by using some relations concerning the spectral norm, Euclidean norm, row norm, column norm. The importance of our results is that our results depend on hyper-Fibonacci and hyper-Lucas numbers. 


\section{CONFLICTS OF INTEREST}

The authors declare that there are no conflicts of interest regarding the publication of this article.

\section{REFERENCES}

[1] Bae, J., Circulant matrix factorization based on schur algorithm for designing optical multimirror filters, Japanese Journal of Applied Physics 45(6A)(2006), 5163-5168. 1

[2] Bahşi, M., On the norms of $r$-circulant matrices with the hyperharmonic numbers, Journal of Mathematical Inequalities 10(2)(2016), 445-458. 1

[3] Bahşi, M., On the norms of circulant matrices with the generalized Fibonacci and Lucas numbers, TWMS J. Pure Appl. Math. 6(1)(2015), 84-92. 1

[4] Bahşi, M., Mezö, I., Solak, S., A symmetric algorithm for hyper-Fibonacci and hyper-Lucas numbers, Annales Mathematicae et Informaticae 43(2014), 19-27. 2, 2

[5] Bahşi, M., S. Solak, On the norms of $r$-circulant matrices with the hyper-Fibonacci and Lucas numbers, Journal of Mathematical Inequalities 8(4)(2014), 693-705. 1

[6] Bahsi, M., Solak, S., On the circulant matrices with arithmetic sequence, Int. J. Cont. Math. Sciences 5(25)(2010), 1213 - 1222.1

[7] Cao, N-N., Zhao, F-Z., Some Properties of Hyperfibonacci and Hyperlucas Numbers, Journal of Integer Sequences 13(2010), Article 10.0.8. 2,2

[8] Davis, P.J., Circulant Matrices, Wiley, New York, Chichester, Brisbane, 1979. 1

[9] Dil, A., Mezö, I., A symmetric algorithm for hyperharmonic and Fibonacci numbers, Appl. Math. Comp. 206(2008), 942-951. 2, 2

[10] Fischer, B., Modersitzki, J., Fast inversion of matrices arising in image processing, Numer. Algorithms 22(1999), 1-11. 1

[11] Georgiou, S.D., Kravvaritis, C., New Good Quasi-Cyclic Codes over GF(3), Int. J. Algebra 1(1)(2007), 11-24. 1

[12] Horn, R.A., Johnson, C.R., Matrix Analysis, Cambridge University Press, Cambridge, 1985. 2.8

[13] Horn, R.A., Johnson, C.R., Topics in Matrix Analysis, Cambridge University Press, Cambridge, 1991. 2.3, 2.4, 2.5, $2.6,2.7$

[14] Karner, H., Schneid, J., Ueberhuber, C.W.,Spectral Decomposition of Real Circulant Matrices, Linear Algebra and Its Appl., 367(2003), 301-311. 1

[15] Kızılateş, C., Tuglu, N., On the bounds for the spectral norms of geometric circulant matrices, Journal of Inequalities and Applications (2016), 2016:312. 1

[16] Kocer, E.G., Circulant, negacyclic and semicirculant matrices with the modified Pell, Jacobsthal and Jacobsthal-Lucas numbers, Hacettepe Journal of Mathematics and Statistics 36(2)(2007), 133-142. 1

[17] Kocer, E.G., Mansour, T., Tuğlu, N., Norms of circulant and semicirculant matrices with Horadam's numbers, Ars Combinatoria 85(2007), 353-359. 1

[18] Tuglu, N., Kızılateş, C., On the norms of circulant and r-circulant matrices with the hyperharmonic Fibonacci numbers, Journal of Inequalities and Applications (2015), 2015:253. 1

[19] Tuglu, N., Kızılateş, C., On the Norms of Some Special Matrices with the Harmonic Fibonacci Numbers, Gazi University Journal of Science 28(3)(2015), 497-501. 1

[20] Öcal, A.A., Tuğlu, N., Altınışık, E., On the representation of k-generalized Fibonacci and Lucas numbers, Appl. Math. Comp. 170(2005), 584-596. 2

[21] Shen, S., Cen, J., On the bounds for the norms of r-circulant matrices with the Fibonacci and Lucas numbers, Appl. Math. Comp. 216(2010), 2891-2897. 1

[22] Solak, S., On the norms of circulant matrices with the Fibonacci and Lucas numbers, Appl. Math. Comp. 160(2005), 125-132. 1

[23] Solak, S., Erratum to "On the Norms of Circulant Matrices with the Fibonacci and Lucas Numbers" [Appl. Math. Comp., 160, (2005), 125-132], Appl. Math. Comp. 190(2007), 1855-1856. 1

[24] Türkmen, R., Gökbaş, H., On the spectral norm of r-circulant matrices with the Pell and Pell-Lucas numbers, Journal of Inequalities and Applications (2016),2016:65. 1

[25] Yazlik, Y., Taskara, N., On the norms of an r-circulant matrix with the generalized k-Horadam numbers, Journal of Inequalities and Applications (2013), 2013:394. 1 